\title{
Assembly of a pan-genome from deep sequencing of 910 humans of African descent
}

\author{
Rachel M. Sherman ${ }^{1,2 \star}$, Juliet Forman ${ }^{1,3}$, Valentin Antonescu', Daniela Puiu', Michelle Daya ${ }^{4}$, \\ Nicholas Rafaels ${ }^{4}$, Meher Preethi Boorgula ${ }^{4}$, Sameer Chavan ${ }^{4}$, Candelaria Vergara ${ }^{5}{ }^{5}$, Victor E. Ortega ${ }^{6}$, \\ Albert M. Levin ${ }^{7}$, Celeste Eng ${ }^{8}$, Maria Yazdanbakhsh ${ }^{9}{ }^{9}$, James G. Wilson ${ }^{10}$, Javier Marrugo", \\ Leslie A. Lange ${ }^{4}$, L. Keoki Williams ${ }^{12}$, Harold Watson ${ }^{13}$, Lorraine B. Ware ${ }^{14}$, Christopher O. Olopade ${ }^{15}$, \\ Olufunmilayo Olopade ${ }^{16}$, Ricardo R. Oliveira ${ }^{17}$, Carole Ober ${ }^{18}$, Dan L. Nicolae ${ }^{16}$, Deborah A. Meyers ${ }^{19}$, \\ Alvaro Mayorga ${ }^{20}$, Jennifer Knight-Madden ${ }^{21}$, Tina Hartert ${ }^{14}$, Nadia N. Hansel ${ }^{5}$, Marilyn G. Foreman ${ }^{22}$, \\ Jean G. Ford ${ }^{23}$, Mezbah U. Faruque ${ }^{24}$, Georgia M. Dunstonn ${ }^{25}$, Luis Caraballo"1, Esteban G. Burchard ${ }^{26}$, \\ Eugene R. Bleecker ${ }^{19}$, Maria I. Araujo ${ }^{27}$, Edwin F. Herrera-Paz ${ }^{28}{ }^{28}$, Monica Campbell ${ }^{4}$, Cassandra Foster ${ }^{5}$, \\ Margaret A. Taub ${ }^{29}$, Terri H. Beaty ${ }^{30}$, Ingo Ruczinski ${ }^{31}$, Rasika A. Mathias ${ }^{5,30}$, Kathleen C. Barnes ${ }^{4}$ and \\ Steven L. Salzberg ${ }^{11,2,29,31 \star}$
}

\begin{abstract}
We used a deeply sequenced dataset of 910 individuals, all of African descent, to construct a set of DNA sequences that is present in these individuals but missing from the reference human genome. We aligned 1.19 trillion reads from the 910 individuals to the reference genome (GRCh38), collected all reads that failed to align, and assembled these reads into contiguous sequences (contigs). We then compared all contigs to one another to identify a set of unique sequences representing regions of the African pan-genome missing from the reference genome. Our analysis revealed $296,485,284$ bp in 125,715 distinct contigs present in the populations of African descent, demonstrating that the African pan-genome contains $-10 \%$ more DNA than the current human reference genome. Although the functional significance of nearly all of this sequence is unknown, 387 of the novel contigs fall within 315 distinct protein-coding genes, and the rest appear to be intergenic.
\end{abstract}

Since its initial publication ${ }^{1,2}$, the human genome sequence has undergone continual improvements aimed at filling gaps and correcting errors. The latest release, GRCh38, spans 3.1 gigabases (Gb), with just 875 remaining gaps ${ }^{3}$. The ongoing effort to improve the human reference genome, led by the Genome Reference Consortium, has in recent years added alternate loci for genomic regions where variation cannot be captured by SNPs or small insertions and deletions (indels). These alternate loci, which comprise 261 scaffolds in GRCh38, capture a small amount of population variation and improve read mapping for some data sets.

Despite these efforts, the current human reference genome derives primarily from a single individual ${ }^{4}$, thus limiting its usefulness for genetic studies, especially among admixed populations, such as those representing the African diaspora. In recent years, a growing number of researchers have emphasized the importance of capturing and representing sequencing data from diverse populations and incorporating these data into the reference genome

\footnotetext{
${ }^{1}$ Center for Computational Biology, McKusick-Nathans Institute of Genetic Medicine, Johns Hopkins University, Baltimore, MD, USA. ${ }^{2}$ Department of Computer Science, Johns Hopkins University, Baltimore, MD, USA. ${ }^{3}$ Departments of Computer Science, Biology, and Mathematics, Harvey Mudd College, Claremont, CA, USA. ${ }^{4}$ Department of Medicine, University of Colorado Denver, Aurora, CO, USA. ${ }^{5}$ Department of Medicine, Johns Hopkins University, Baltimore, MD, USA. ${ }^{6}$ Department of Internal Medicine, Section on Pulmonary, Critical Care, Allergy and Immunologic Diseases, Center for Precision Medicine, Wake Forest School of Medicine, Winston-Salem, NC, USA. 'Department of Public Health Sciences, Henry Ford Health System, Detroit, MI, USA. ${ }^{8}$ Department of Medicine, University of California, San Francisco, San Francisco, CA, USA. 'Department of Parasitology, Leiden University Medical Center, Leiden, The Netherlands. ${ }^{10}$ Department of Physiology and Biophysics, University of Mississippi Medical Center, Jackson, MS, USA. "'Institute for Immunological Research, Universidad de Cartagena, Cartagena, Colombia. ${ }^{2}$ Department of Internal Medicine, Henry Ford Health System, Detroit, MI, USA. ${ }^{13}$ Faculty of Medical Sciences Cave Hill Campus, The University of the West Indies, Bridgetown, Barbados. ${ }^{14}$ Department of Medicine, Vanderbilt University, Nashville, TN, USA. ${ }^{15}$ Department of Medicine and Center for Global Health, University of Chicago, Chicago, IL, USA. ${ }^{16}$ Department of Medicine, University of Chicago, Chicago, IL, USA. ${ }^{17}$ Laboratório de Patologia Experimental, Centro de Pesquisas Gonçalo Moniz, Salvador, Brazil. ${ }^{18}$ Department of Human Genetics, University of Chicago, Chicago, IL, USA. ${ }^{19}$ Department of Medicine, University of Arizona College of Medicine, Tucson, AZ, USA. ${ }^{20} \mathrm{C}$ entro de Neumologia y Alergias, San Pedro Sula, Honduras. ${ }^{21}$ Caribbean Institute for Health Research, The University of the West Indies, Kingston, Jamaica. ${ }^{22}$ Pulmonary and Critical Care Medicine, Morehouse School of Medicine, Atlanta, GA, USA. ${ }^{23}$ Department of Medicine, Einstein Medical Center, Philadelphia, PA, USA. ${ }^{24}$ National Human Genome Center, Howard University College of Medicine, Washington, DC, USA. ${ }^{25}$ Department of Microbiology, Howard University College of Medicine, Washington, DC, USA. ${ }^{26}$ Departments of Bioengineering \& Therapeutic Sciences and Medicine, University of California, San Francisco, San Francisco, CA, USA. ${ }^{27}$ Immunology Service, Universidade Federal da Bahia, Salvador, Brazil. ${ }^{28}$ Facultad de Ciencias Médicas, Universidad Tecnológica Centroamericana (UNITEC), Tegucigalpa, Honduras. ${ }^{29}$ Department of Biostatistics, Bloomberg School of Public Health, Johns Hopkins University, Baltimore, MD, USA. ${ }^{30}$ Department of Epidemiology, Bloomberg School of Public Health, Johns Hopkins University, Baltimore, MD, USA. ${ }^{31}$ Department of Biomedical Engineering, Johns Hopkins University, Baltimore, MD, USA. *e-mail: rsherman@jhu.edu; salzberg@jhu.edu
} 
and genomics studies in general ${ }^{5-7}$. The alternate loci in GRCh38 offer one possible way to add such diversity, although it is unclear whether such a solution is sustainable as more populations are sequenced. Among other problems, the addition of alternate loci as separate contigs can mislead sequence alignment programs, which were designed under the assumptions that each read has a single true point of origin and that the genome is represented as a linear haploid sequence ${ }^{8}$.

The lack of diversity in the reference genome poses many challenges when analyzing individuals whose genetic background does not match the reference. This problem may be addressed by using large databases of known SNPs (for example, dbSNP ${ }^{9}$ ), but this solution only addresses single-base differences and small indels and is not adequate for larger variants. Findings from the 1000 Genomes Project indicate that differences between populations are quite large; examination of 26 populations across five continents revealed that $86 \%$ of discovered variants were present in only one continental group. In that study, the five African populations examined had the highest number of variant sites compared with the remaining 21 populations ${ }^{10}$.

One way to address the limitations of a single reference genome is to sequence and assemble reference genomes for other human subpopulations. The 1000 Genomes Project, Genome in a Bottle, and other projects have assembled draft genomes from various populations, including Chinese, Korean, and Ashkenazi individu$\mathrm{als}^{11-15}$. Other groups have used highly homogenous populations (for example, Danish, Dutch, or Icelandic individuals) together with assembly-based approaches to discover SNPs and structural variants (SVs), including up to several megabases of non-reference sequence common to these populations ${ }^{16-19}$. Although these variant analyses are a step in the right direction, to date, none have produced a reference-quality genome that can replace GRCh38 (ref. ${ }^{3}$ ); however, this is an explicit goal of the Danish Genome Project (URLs).

While efforts to produce new reference genomes are worthwhile, attempts to create a pan-genome of a human population, a collection of sequences representing all of the DNA in that population, are rare. Although multiple pan-genomes have been created for bacterial species ${ }^{20-22}$, as of yet, there are no reported pan-genomes for any other animal species, to our knowledge. The lack of pan-genomes is due in part to the technical challenges of assembling many deeply sequenced genomes de novo and combining them into one genome. Whereas the Danish Genome Project focused on 50 trios of non-admixed individuals (removing admixed samples from their study ${ }^{17}$ ), our study focuses on a highly heterogeneous group of admixed individuals. Because the human reference genome is largely complete (the sequence has very few gaps), our strategy for creating a pan-genome focused on finding large insertions. This approach, although computationally demanding, made the African pan-genome assembly process described here feasible.

A 2010 study that sequenced one Asian and one African individual used the novel sequences identified to estimate that a full human pan-genome would contain an additional 19-40 megabases $(\mathrm{Mb})$ that are not in the current reference genome ${ }^{11}$. Recent efforts to sequence a Dutch population and a set of 10,000 individuals have supported this estimate, reporting 4.3 and $3.3 \mathrm{Mb}$ of nonreference sequences, respectively ${ }^{18,23}$; however, neither study was designed with the primary goal of discovering long, non-reference sequences. A 2017 study in which two haploid human genomes (hydatidiform moles) were sequenced using long reads estimated that a single diploid genome may differ by as much as $16 \mathrm{Mb}$ from the reference genome ${ }^{24}$. As we describe here, our analysis of 910 deeply sequenced individuals, all from the Consortium on Asthma among African-Ancestry Populations in the Americas (CAAPA) ${ }^{25}$, produced a much larger amount of novel sequence (sequence absent from GRCh38) in the African pan-genome, spanning 296.5 Mb. We describe the methods used to identify and validate these sequences along with comparisons to other human sequences. The African pan-genome (APG) contigs have been deposited at NCBI under accession PDBU01000000 to provide a better foundation for future analyses of individuals of African ancestry.

In total, we discovered $296.5 \mathrm{Mb}$ of novel DNA distributed across 125,715 sequences assembled from 910 individuals of African descent (Table 1 and Supplementary Fig. 1). We took steps to ensure contaminants and redundant contigs were removed, resulting in a non-redundant set of human contigs representative of the entire study group (Fig. 1). After discovery, we called presence/absence for all APG sequences in each CAAPA sample. A total of 33,599 contigs with a combined length of $81,096,662$ bases represented sequences present in at least two individuals in the CAAPA cohort. When alignments above $80 \%$ coverage and $90 \%$ identity to Chinese and Korean genome assemblies were also considered shared, the number of non-private insertions increased to 61,410 , totaling $160,475,353$ bases and leaving 64,305 singleton contigs, a $\sim 51 \%$ singleton rate. Of the 125,715 APG sequences, 1,548 (total length $4.4 \mathrm{Mb}$ ) were anchored to a specific location in the primary GRCh38 assembly. On average, each individual contained 859 of these inserted sequences, with a single sequence being shared among six individuals (Table 2). Placed contigs were shared among more individuals, 196 on average, as shared sequences were more likely to meet the placement criterion in at least one individual.

We fully resolved the location for 302 of these sequences and resolved the breakpoint of one end of the insertion for the remaining 1,246 (Supplementary Table 1). Placement locations were determined by complementing our methods with results from the PopIns program $^{16}$, which corroborated many placements and resolved placements for some insertions for which our method was ambiguous (Supplementary Note 1). The remaining sequences (Supplementary Table 2) could not be fully localized; however, mate-linking information pointed to a consistent location for at least one end for an additional 57,655 sequences (Supplementary Table 3). The longest placed sequence was 79,938 bp and appeared in 197 samples, and the longest unplaced sequence was $152,806 \mathrm{bp}$, which appeared in 11 samples (Table 1). Among all placed sequences, 387 intersected known genes, with placements within exons in 48 distinct genes and placements within introns in an additional 267 genes (some genes contained more than one insertion). Of the 315 genes containing insertions, 292 were named (had names other than 'hypothetical' or a non-meaningful identifier). An additional 133 placed insertions and 46 that already intersected a protein-coding gene intersected 142 distinct lncRNAs, 21 of which were named (Supplementary Table 4). A translated BLAST $^{26}$ search on unplaced sequences against NCBI's nr database yielded an additional 10,667 contigs hitting a chordate protein with $\geq 70 \%$ identity and an e value less than

\begin{tabular}{|c|c|c|c|c|}
\hline & $\begin{array}{l}\text { Number of } \\
\text { sequence } \\
\text { contigs }\end{array}$ & $\begin{array}{l}\text { Total length } \\
\text { (bp) }\end{array}$ & $\begin{array}{l}\text { Bases with no } \\
\text { alignment to } \\
\text { GRCh38 ( }<80 \% \\
\text { identity) }\end{array}$ & $\begin{array}{l}\text { Longest } \\
\text { contig } \\
\text { (bp) }\end{array}$ \\
\hline Two ends placed & 302 & 667,668 & 431,656 & 20,732 \\
\hline One end placed & 1,246 & $3,687,028$ & $1,866,699$ & 79,938 \\
\hline Unplaced & 124,167 & $292,130,588$ & $202,629,979$ & 152,806 \\
\hline Total & 125,715 & $296,485,284$ & $204,928,334$ & 152,806 \\
\hline Non-private only & 33,599 & $80,098,092$ & $50,044,650$ & 152,806 \\
\hline
\end{tabular}

Number and length of novel sequences in the African pan-genome. Bases with no alignment to GRCh38 were calculated by subtracting the lengths of all subsequences that aligned with at least $80 \%$ identity. The remainder represents truly novel sequence. Non-private insertions were insertions shared by at least two CAAPA cohort individuals. 


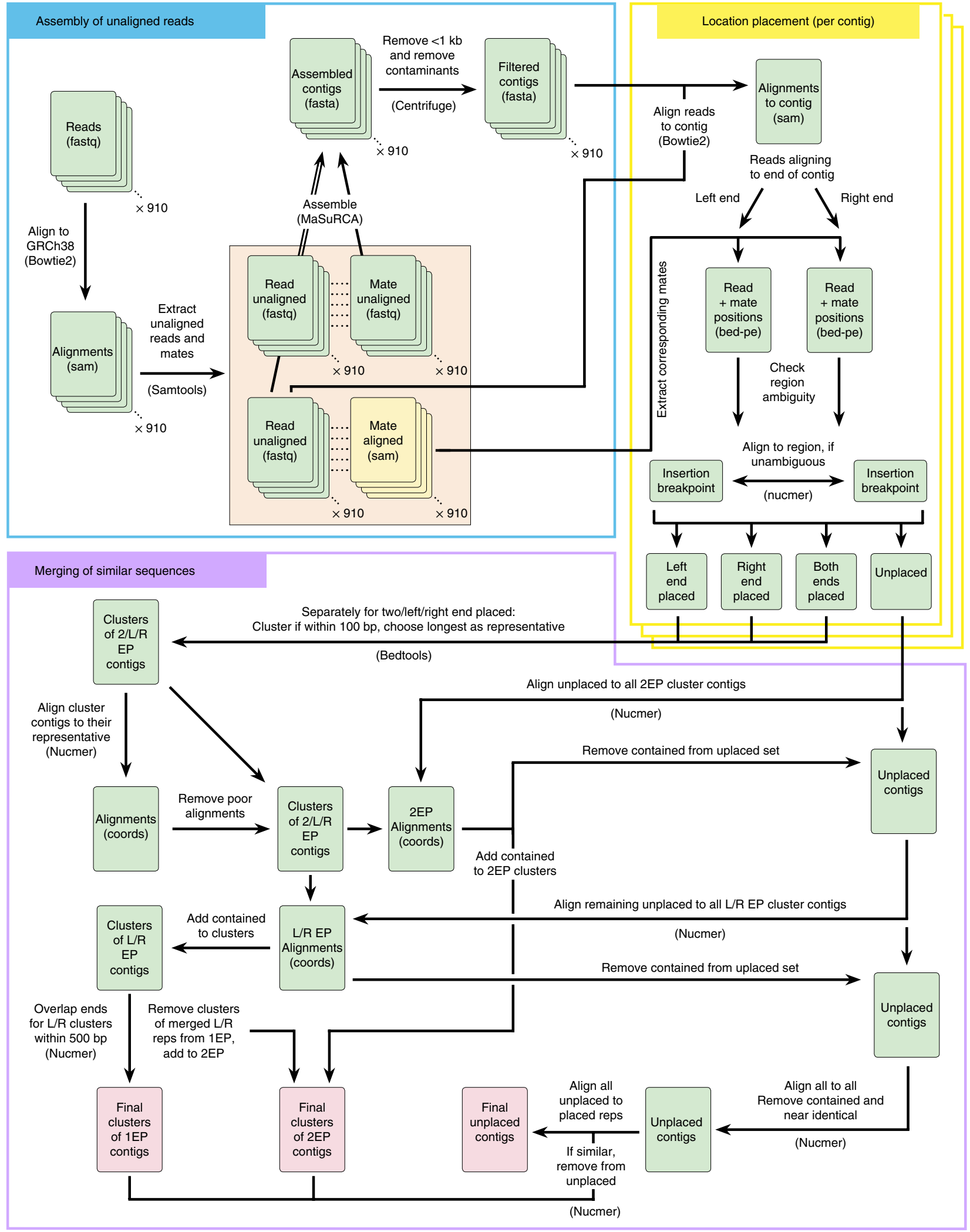

Fig. 1 | Overview of methods. Raw reads are aligned to GRCh38, and unaligned reads are assembled with MaSuRCA. Assembled contigs are then filtered for contaminants with Centrifuge, and contigs shorter than $1 \mathrm{~kb}$ are removed (blue box). Assembled contigs are placed based on their mate's alignment locations when possible by checking whether $>95 \%$ of mates align to the same location. If such a placement is found, the exact breakpoint is determined via a nucmer alignment to the region for each end of the contig (yellow box). Contig placement locations are then compared between all individuals, nearby placements are clustered, and a representative is chosen. All contigs are then aligned to the representatives to determine which samples contain a given placed insertion. Contigs in or aligning to placed clusters are removed from the unplaced set, and the remaining unplaced contigs are aligned to one another with nucmer to remove redundancy and result in a final nonredundant unplaced set of contigs (purple box). EP, end placed; 1EP, one end placed; $2 E P$, two end placed; L, left; R, right. 

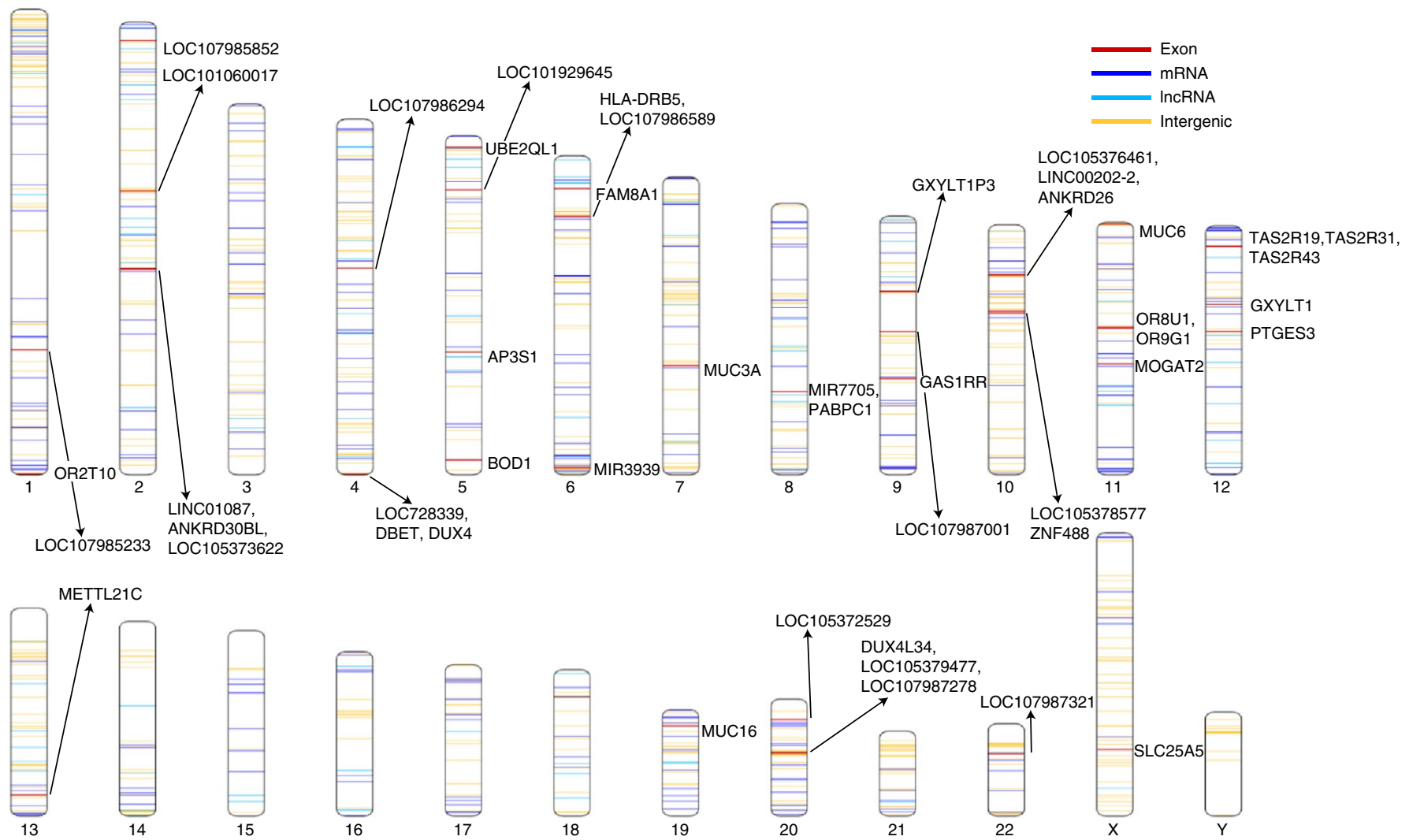

Fig. 2 | African pan-genome contig locations. Map of the human genome showing the locations of all African pan-genome contigs, for those that could be placed accurately along one of the chromosomes. Yellow lines represent an intergenic location; blue lines represent insertion points with RNA but not exonic annotations, and red lines indicate intersections within exons. All exon-intersecting insertions are labeled with the gene name. mRNA and IncRNA gene names are reported in Supplementary Table 4. In some cases, insertions are too close together for lines to be resolved; when this occurs within exons, gene names are listed in order by chromosome position. Line width is not to scale.

\begin{tabular}{|c|c|c|c|}
\hline & $\begin{array}{l}\text { Number of } \\
\text { contigs }\end{array}$ & $\begin{array}{l}\text { Mean number } \\
\text { of insertions per } \\
\text { individual }\end{array}$ & $\begin{array}{l}\text { Mean number } \\
\text { individuals per } \\
\text { insertion }\end{array}$ \\
\hline Two ends placed & 302 & $120(39.7 \%)$ & 363 (of 910) \\
\hline One end placed & 1,246 & $212(17.0 \%)$ & 155 (of 910) \\
\hline Unplaced & 124,167 & $527(0.4 \%)$ & 4 (of 910) \\
\hline Total & 125,715 & $859(0.7 \%)$ & 6 (of 910) \\
\hline Non-private only & 33,599 & $758(2.2 \%)$ & 21 (of 910) \\
\hline
\end{tabular}

Statistics on the presence or absence of the African pan-genome contigs. Presence/absence was determined by aligning all raw contigs for each individual to the final set of APG contigs. Alignments of one or more contigs yielded a presence call if the alignments covered at least $80 \%$ of an APG contig at at least $90 \%$ identity. Additional presence calls were made for the placed contigs if the individual had a similar contig placed in the same location, even if the alignment thresholds were not met.

$1 \times 10^{-10}$. Placement locations and gene intersections were dispersed throughout the genome, and placed pan-genome elements were found on every chromosome (Fig. 2), in addition to 115 insertions in chromosome-specific 'random' sequences and 103 more in 'unlocalized' sequences included in the primary assembly of GRCh38.

Of our APG contigs, 31,354,079 bases aligned to a GRCh38 'patch' or alternate (ALT) locus as part of an alignment with an identity of $\geq 80 \%$. An additional $60,202,871$ bases aligned to the primary assembly at $\geq 80 \%$ identity; however, most of these alignments covered a small portion of an APG contig and can be explained by the presence of extra copies of small repetitive elements. Data in Supplementary Tables 1 and 2 report alignments to ALT, patch, or primary assembly sequences covering at least $50 \%$ of the contig length with $\geq 80 \%$ identity. Requiring that at least $50 \%$ of a contig be aligned to any single location in GRCh 38 produced a much smaller subset: of the 125,715 contigs, only 17,140 aligned to any part of GRCh38.p10 with a single alignment at $\geq 80 \%$ identity covering $\geq 50 \%$ of the contig length. These 17,140 contigs contain $22,420,979$ aligned bases, with $13,770,950$ bases being alignments to a reference chromosome. Although very few ALT loci in GRCh38.p10 are tagged with population-specific information, alignments of the CAAPA-specific sequences to these loci suggest an African source for some of these ALT sequences.

In addition to calling presence/absence of our APG insertions in the CAAPA individuals, we performed a similar analysis of 12 European and 12 African individuals from the Simons Genome Diversity Project (SGDP) ${ }^{27}$. The SGDP individuals varied in the number of APG sequences they contained (Supplementary Table 5), though analyzing the European- versus African-only contigs demonstrated that the APG insertions tend to be more representative of African than European assemblies, despite the admixed nature of the data (Supplementary Note 2).

We additionally aligned all 125,715 pan-genome contigs to recent human assemblies of Chinese (HX1) ${ }^{14}$ and Korean (KOREF1.0) ${ }^{15}$ individuals using bwa-mem ${ }^{28}$. We detected 42,207 contigs totaling $120.7 \mathrm{Mb}$ aligning to either the Korean or Chinese assembly's with $\geq 90 \%$ identity and $\geq 80 \%$ contig coverage, and matching the Chinese or Korean assembly better than GRCh38. A vast majority of these contigs $(32,955)$ had no alignment at $\geq 80 \%$ identity and 
Table 3 | Comparison of African pan-genome contigs to the Chinese and Korean genomes

\begin{tabular}{lllllll} 
& \multicolumn{2}{l}{$\begin{array}{l}\text { Best GRCh38 alignment is } \mathbf{8 0 - 9 0 \%} \\
\text { identical with }\end{array}$} & \multicolumn{2}{l}{$\begin{array}{l}\text { Best GRCh38 alignment is }<\mathbf{8 0 \%} \text { coverage } \\
\text { identical or }<\mathbf{5 0 \%} \text { coverage }\end{array}$} & Total \\
\cline { 2 - 7 } & Contigs & Length (bp) & Contigs & Length (bp) & Contigs & Length (bp) \\
\hline Matches Chinese only & 1,625 & $2,898,106$ & 7,607 & $25,475,277$ & 9,232 & $28,373,383$ \\
Matches Korean only & 2,242 & $3,989,277$ & 15,635 & $48,642,664$ & 17,877 & $52,631,941$ \\
Matches both & 5,385 & $9,720,662$ & 9,713 & $29,981,048$ & 15,098 & $39,701,710$ \\
Total & 9,252 & $16,608,045$ & 32,955 & $104,098,989$ & 42,207 & $120,707,034$ \\
\hline
\end{tabular}

Contigs with a better alignment to the Chinese or Korean assemblies than to GRCh38. Alignments to the Chinese and Korean assemblies were required to have $\geq 90 \%$ identity and $\geq 80 \%$ coverage to be considered. Lengths shown are the sums of the contig lengths, not the alignment lengths.

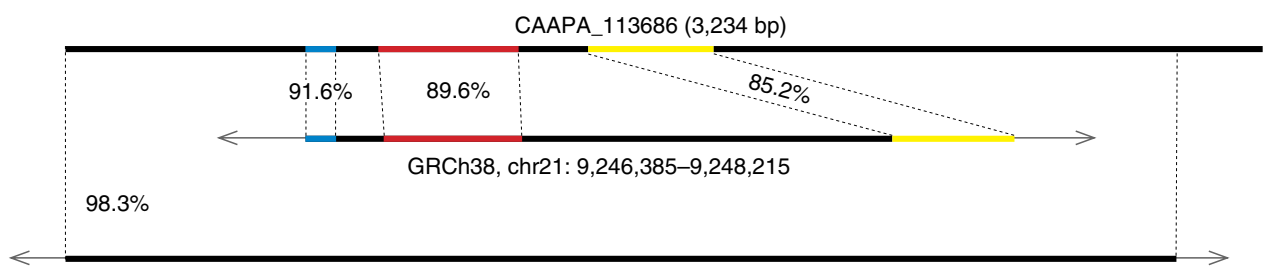

Chinese HX1, 005482F: 84-3,084

Fig. 3 | An example of an alignment that does not meet the $50 \%$ coverage, $80 \%$ identity threshold for a 'reasonably good' alignment to GRCh38. The APG contig is shown at the top, with the best consistent alignments to GRCh38 in the middle. The three constituent alignments (blue, red, and yellow segments) cover 801 bases, just under 25\% of the contig, with a cumulative weighted identity of 87.9\%. CAAPA_113686 has a single near-perfect alignment to a Chinese HX1 contig (delineated by dotted lines) covering over $80 \%$ of CAAPA_ 113686 at over $90 \%$ identity. The APG contig also aligns well to the Korean assembly (not shown).

$\geq 50 \%$ coverage to GRCh38.p10, indicating that these sequences were not simply divergent from GRCh38, but rather were not present at all (Table 3); an example of such a sequence and its alignments to GRCh38 and HX1 are shown in Fig. 3. This finding suggests these sequences have been lost in the small number of individuals used to create GRCh38, although some of them may reside in the few remaining gaps in the genome.

While Shi et al. reported $12.8 \mathrm{Mb}$ of novel DNA in the HX1 genome $^{14}$, we found a total of $68.1 \mathrm{Mb}$ shared by HX1 and the unique sequences in the APG contigs (Table 3). This discrepancy is methodological: the Chinese genome assembly has relatively large scaffolds that were considered unique only if a large proportion of the scaffold failed to align to GRCh38 (Supplementary Note 3).

As an additional check to ensure the APG sequences were not contaminants, we examined what portion of contigs had some match, even just a partial match, to the GRCh38, Korean, or Chinese assemblies. After filtering to retain only query-consistent alignments, $98 \%$ of the contigs $(123,600)$ had some portion aligning to either the Chinese, Korean, or GRCh38 assemblies. The Korean assembly had the most alignment, with 123,585 contigs containing an alignment totaling $247.2 \mathrm{Mb}$ of aligned length, or $83 \%$ of the total APG sequence, although only 31,033 contigs, totaling $80.9 \mathrm{Mb}$ of alignment, aligned with over $\geq 90 \%$ identity and $\geq 80 \%$ coverage.

Our findings here demonstrate that the standard human reference genome lacks a substantial amount of DNA sequence compared with other human populations. The APG sequences contain $296.5 \mathrm{Mb}$, equal to $10 \%$ of the genome, regions that will necessarily be missed by any efforts relying only on GRCh38 to study human variation, as nearly all studies do at present. Of these $296.5 \mathrm{Mb}, 120.7 \mathrm{Mb}$ were shared by the Korean or Chinese populations, suggesting those regions may have been lost more recently or may be rare in the specific populations represented in GRCh38. Overall these results suggest that a single reference genome is not adequate for population-based studies of human genetics. Instead, a better approach may be to create reference genomes for all distinct human populations, which over time will eventually yield a comprehensive pan-genome capturing all of the DNA present in humans.

URL. http://www.genomedenmark.dk/english/about/referencegenome/.

\section{Online content}

Any methods, additional references, Nature Research reporting summaries, source data, statements of data availability and associated accession codes are available at https://doi.org/10.1038/ s41588-018-0273-y.

Received: 20 November 2017; Accepted: 8 October 2018; Published online: 19 November 2018

\section{References}

1. International Human Genome Sequencing Consortium. Initial sequencing and analysis of the human genome. Nature 409, 860-921 (2001).

2. Venter, J. C. et al. The sequence of the human genome. Science 291, 1304-1351 (2001).

3. Schneider, V. A. et al. Evaluation of GRCh38 and de novo haploid genome assemblies demonstrates the enduring quality of the reference assembly. Genome Res. 27, 849-864 (2017).

4. Green, R. E. et al. A draft sequence of the Neandertal genome. Science 328 710-722 (2010).

5. E pluribus unum. Nat Methods 7, 331 (2010).

6. Need, A. C. \& Goldstein, D. B. Next generation disparities in human genomics: concerns and remedies. Trends Genet. 25, 489-494 (2009).

7. Popejoy, A. B. \& Fullerton, S. M. Genomics is failing on diversity. Nature 538, 161-164 (2016)

8. Church, D. M. et al. Extending reference assembly models. Genome. Biol. 16, 13 (2015).

9. Sherry, S. T. et al. dbSNP: the NCBI database of genetic variation. Nucleic Acids Res. 29, 308-311 (2001).

10. The 1000 Genomes Project Consortium. A global reference for human genetic variation. Nature 526, 68-74 (2015).

11. Li, R. et al. Building the sequence map of the human pan-genome. Nat. Biotechnol. 28, 57-63 (2010). 
12. Seo, J. S. et al. De novo assembly and phasing of a Korean human genome. Nature 538, 243-247 (2016).

13. Zook, J. M. et al. Extensive sequencing of seven human genomes to characterize benchmark reference materials. Sci. Data 3, 160025 (2016).

14. Shi, L. et al. Long-read sequencing and de novo assembly of a Chinese genome. Nat. Commun. 7, 12065 (2016).

15. Cho, Y. S. et al. An ethnically relevant consensus Korean reference genome is a step towards personal reference genomes. Nat. Commun. 7, 13637 (2016).

16. Kehr, B., Melsted, P. \& Halldorsson, B. V. PopIns: population-scale detection of novel sequence insertions. Bioinformatics 32, 961-967 (2016).

17. Maretty, L. et al. Sequencing and de novo assembly of 150 genomes from Denmark as a population reference. Nature 548, 87-91 (2017).

18. Hehir-Kwa, J. Y. et al. A high-quality human reference panel reveals the complexity and distribution of genomic structural variants. Nat. Commun. 7, 12989 (2016).

19. Kehr, B. et al. Diversity in non-repetitive human sequences not found in the reference genome. Nat. Genet. 49, 588-593 (2017).

20. Gordienko, E. N., Kazanov, M. D. \& Gelfand, M. S. Evolution of pan genomes of Escherichia coli, Shigella spp., and Salmonella enterica. J. Bacteriol. 195, 2786-2792 (2013).

21. Tettelin, H. et al. Genome analysis of multiple pathogenic isolates of Streptococcus agalactiae: implications for the microbial "pan-genome". Proc. Natl Acad. Sci. USA 102, 13950-13955 (2005).

22. Vernikos, G., Medini, D., Riley, D. R. \& Tettelin, H. Ten years of pan-genome analyses. Curr. Opin. Microbiol. 23, 148-154 (2015).

23. Telenti, A. et al. Deep sequencing of 10,000 human genomes. Proc. Natl Acad. Sci. USA 113, 11901-11906 (2016).

24. Huddleston, J. et al. Discovery and genotyping of structural variation from long-read haploid genome sequence data. Genome Res. 27, 677-685 (2017).

25. Mathias, R. A. et al. A continuum of admixture in the Western Hemisphere revealed by the African Diaspora genome. Nat. Commun. 7, 12522 (2016).

26. Camacho, C. et al. BLAST+: architecture and applications. BMC Bioinformatics 10, 421 (2009).

27. Mallick, S. et al. The Simons Genome Diversity Project: 300 genomes from 142 diverse populations. Nature 538, 201-206 (2016).

28. Li, H. \& Durbin, R. Fast and accurate short read alignment with BurrowsWheeler transform. Bioinformatics 25, 1754-1760 (2009).

\section{Acknowledgements}

We gratefully acknowledge the contributions of P. Maul, T. Maul, P. Levett, A. Hennis, P. M. Lashley, R. Naidu, M. Howitt, T. Roach, A. Grant, E. V. Ponte, A. A. Cruz, E. Carvalho, S. Balcer-Whaley, M. Stockton-Porter, M. Yang, D. Mejía-Mejía, M. Meraz, J. Nuñez, E. F. Herrera Mejía, T. Ferguson, D. Ashley, S. Jimenez, N. Acevedo, D. Mercado, A. Jedlicka, H. Gui, R. Torres, A. K. May, C. Gilmore, P. Minton, Q. Niu, A. Falusi, and A. Odetunde. The authors also acknowledge the support of J. J. Shannon, K. Weiss, R. Miranda and the Indians Zenues guards, U. A. Ngoa, C. Rotimi, A. Adebowale, F. J. Malveaux, and E. Reece. We thank the numerous healthcare providers, community clinics and coinvestigators who assisted in the phenotyping and collection of DNA samples, and the families and patients for generously donating DNA samples to BAGS, BIAS, BREATHE, CAG, GRAAD, HONDAS, Jackson Heart Study, REACH, SAGE II, VALID, SAPPHIRE, SARP, COPDGene, JAAS, GALA II, PGCA, AEGS, and the asthma studies in Gabon and Palenque, Colombia. Special thanks to community leaders, teachers, doctors and personnel from health centers at the Garifuna communities for organizing the medical brigades and to the medical students at Universidad Católica de Honduras, Campus San Pedro y San Pablo for their participation in the fieldwork related to HONDAS; study coordinator S. Salazar, and the recruiters in SAGE and GALA: D. Alva, G. Ayala-Rodriguez, U. Burley, L. Caine, E. Castellanos, J. Colon, D. DeJesus, I. Flexas, B. Lopez, B. Lopez, L. Martos, V. Medina, J. Olivo, M. Peralta, E. Pomares, J. Quraishi, J. Rodriguez, S. Saeedi, D. Soto, A. Taveras, E. Viera, M. LeNoir, K. Meade, M. Jensen, and A. Davis; and health liaisons and public health officers of the main Conde office, A. Conceição, L. Quintela, I. Santos, A. Lima, B. Valber Oliveira Silva, and I. S. Araujo, and students from the Federal University of Bahia who assisted in data collection in BIAS: R. Santana, R. Barbosa, A. P. Santana, C. Barros, M. Brandão, L. Almeida, T. Cardoso, and D. Costa. We are grateful for the support from the international state governments and universities from Honduras, Colombia, Brazil, Gabon, Nigeria, The Netherlands, Jamaica, Barbados, and the United States, who made this work possible. Funding for this study was provided in part by NIH grants R01-HL129239 (S.L.S. and K.C.B.), R01-HG006677 (S.L.S.), and R01HL104608 (K.C.B. and R.A.M.).

\section{Author contributions}

R.M.S. designed and performed analyses and wrote the paper. J.F. performed analyses. V.A preprocessed data. D.P. performed analyses. M.D. collected data and provided comments on the manuscript. N.R., M.P.B., S.C., C.V., V.E.O., A.M.L., C.E., M.Y., J.G.W., J.M., L.A.L., L.K.W., H.W., L.B.W., C.O.O., O.O., R.R.O., C.O., D.L.N., D.A.M., A.M., J.K.-M., T.H., N.N.H., M.G.F., J.G.F., M.U.F., G.M.D., L.C., E.G.B., E.R.B., M.I.A., E.F.H.-P., M.C., and C.F. collected data. M.A.T., T.H.B., and I.R. collected data and provided comments on the manuscript. R.A.M. collected data. K.C.B. collected data and provided comments on the manuscript. S.L.S. conceived and advised the project and wrote the paper.

\section{Competing interests}

The authors declare no competing interests.

\section{Additional information}

Supplementary information is available for this paper at https://doi.org/10.1038/ s41588-018-0273-y.

Reprints and permissions information is available at www.nature.com/reprints. Correspondence and requests for materials should be addressed to R.M.S. or S.L.S Publisher's note: Springer Nature remains neutral with regard to jurisdictional claims in published maps and institutional affiliations.

(c) The Author(s) 2018

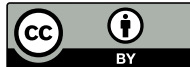

Open Access This article is licensed under a Creative Commons Attribution 4.0 International License, which permits use, sharing, adaptation, distribution and reproduction in any medium or format, as long as you give appropriate credit to the original author(s) and the source, provide a link to the Creative Commons license, and indicate if changes were made. The images or other third party material in this article are included in the article's Creative Commons license, unless indicated otherwise in a credit line to the material. If material is not included in the article's Creative Commons license and your intended use is not permitted by statutory regulation or exceeds the permitted use, you will need to obtain permission directly from the copyright holder. To view a copy of this license, visit http://creativecommons.org/licenses/by/4.0/. 


\section{Methods}

We used whole-genome shotgun sequence data from 910 individuals whose genomes were sequenced as part of the CAAPA project, available from $\mathrm{dbGaP}$ as accession phs001123.v1.p1. The total data set contains 1.19 trillion $\left(1.19 \times 10^{12}\right)$ 100 -bp paired end reads, representing an average of 30-40× coverage for each individual's genome. Sequencing was performed on an Illumina HiSeq 2000. The subjects in the study were all of African ancestry and were selected from 19 populations across the Americas, the Caribbean, and continental Africa ${ }^{25}$ (Supplementary Table 6).

Assembly of novel contigs. For each sample, we aligned all reads to GRCh38. p0 using Bowtie 2 (ref. ${ }^{29}$ ) and extracted unaligned reads and their mates using Samtools ${ }^{30}$ (Fig. 1). GRCh38 alternate loci were excluded from the reference index, but were considered later in the process. We then assembled all unaligned reads with the MaSuRCA assembler ${ }^{31}$; if neither mate in a pair aligned to GRCh38, MaSuRCA treated the reads as paired ends with a fragment size of $300 \mathrm{bp}$, and if only one mate was unaligned, MaSuRCA treated it as an unpaired read.

We filtered the resulting assemblies to exclude contigs shorter than $1000 \mathrm{bp}$ (Fig. 1) and evaluated all remaining contigs with the Centrifuge metagenomics program $^{32}$, scanning against the comprehensive NCBI nucleotide database to obtain a taxonomic classification of each contig. We considered any contigs labeled by Centrifuge as non-chordates (for example, bacterial or viral contigs) to be contaminants and removed them from further consideration.

Positioning contigs within GRCh38. We attempted to place the assembled contigs in a precise location in the human genome using mapping information from paired reads ("mates"). We masked contigs with RepeatMasker ${ }^{33}$ with the lowcomplexity option off (-nolow) and used Bowtie 2 to realign all unaligned reads from read pairs in which only one mate had aligned originally. For each read $R$ aligning within 500 bases of the end of a contig, we examined the alignment of $R$ 's mate to GRCh38 to determine whether the contig had a unique placement in the reference genome. The fragment length for all paired-end libraries was $300 \mathrm{bp}$; by considering reads within $500 \mathrm{bp}$ of the end of a contig, we reduced the likelihood that one or both of the alignments was a spurious match. Additional details of the sequencing protocols for the CAAPA genomes are described elsewhere ${ }^{25}$. This process resulted in a pool of linking mates corresponding to the beginning and end of each contig.

We then separated contigs into several groups based on their linking information:

1. No linking mates existed on either end of the contig; the reads mates did not align to GRCh38.

2. Placement was unambiguous (or unique) for at least one end of the contig. We define 'chromosome unambiguous' to mean $>95 \%$ of the linking mates linked to the same chromosome. We define 'region unambiguous' to mean that of the $>95 \%$ of mates aligned to the same chromosome, all mates aligned within $2 \mathrm{~kb}$ of each other. When both conditions hold, we say placement is unambiguous. These contigs were further divided into two subgroups:

a. Both ends of the contig were placed unambiguously, or

b. Only one end was placed unambiguously.

3. At least one end of the contig was chromosome unambiguous, but neither end was region unambiguous.

4. Neither end was chromosome unambiguous.

For all contigs in the second group, we used NUCmer ${ }^{34}$ to align them to the region determined by the linking mates (Fig. 1). If a contig end had one or more consistent exact matches of at least 15 bases (and no inconsistent alignments), we then determined the contig end's exact insertion location based on alignment coordinates (Supplementary Methods). We permitted an exact two-ended placement only if both ends aligned to the same reference region with the same orientation. The insertion position was either a single breakpoint, if both ends of the contig were placed identically, or a range, if the insertion location of the two ends was not identical. For contigs with only a single end exactly placed, we recorded their exact single-end insertion position and the number of overlapping bases (bases to be trimmed off the end of the contig).

Insertion discovery with PopIns. To supplement the list of placed contigs determined by the procedure above, we ran the PopIns program ${ }^{16}$, which was used previously for a set of genomes from Icelandic individuals, and was designed to find insertions from a relatively genetically homogenous population. We ran PopIns beginning with the popins merge step, using the cleaned MaSuRCA contig assemblies described above. We ran subsequent PopIns steps as recommended in the PopIns documentation, through the popins place-finish step. PopIns output was converted into a comparable format, and verifiable placements were added to our sets of insertions (Supplementary Methods).

Clustering of placed contigs. Once contig locations were determined for each individual sample, we aligned all insertions to one another and clustered them to determine which contigs represented the same insertion across individuals (Fig. 1).
Clustering two-ended placements. For contigs with both ends placed, we ran BEDtools merge ${ }^{35}$ to group contigs placed at approximately the same location. We used the -d option with a distance of 10 to allow placements within 10 bases of each other to be combined. We also ran the merge using -d 100, which produced identical results. For each resulting region and contig cluster, we chose the longest contig in the cluster as the cluster's representative $(R)$, and these representatives formed the initial set of two-end placed contigs, 2EP. Two-ended placement clusters from PopIns were then added to 2EP. We verified clusters by aligning all contigs in each cluster to its representative, $R$, with default nucmer parameters and removing from the cluster any contigs that did not have any alignments to $R$. To find the complete set of samples containing each insertion, we then aligned all remaining contigs (including unplaced contigs) to the contigs in the clusters. Any contig aligning with $>99 \%$ identity that was fully contained within a contig in a cluster $C$ and covered $\geq 80 \%$ of the contig in $C$ was included in $C$ as part of the final set. Contained, $99-100 \%$ identical contigs aligning with $<80 \%$ coverage were also included if they had at least five linking mates and at least $25 \%$ of those mates linked to within $5 \mathrm{~kb}$ of the placement location. The longest representative contig in each cluster was used as the final insertion sequence for the African Pan-Genome (APG) contig collection (Supplementary Tables 1 and 2).

Clustering one-ended placements. We separated contigs with only one end placed into two groups: (1) contigs where the "left" end aligned to the reference, so that the contig extends into a gap to the right of the placement location; and (2) contigs with their "right" end placed, so the contig extends into a gap to the left of the placement location (Fig. 1). Left and right were determined by the orientation of the chromosomes in GRCh38. We then created clusters separately for the two groups using BEDtools merge (-d 100) as described above, identifying the longest representative $R$ for each group. This formed the initial set of one-end placed contigs, 1EP. Any placements within 100 bases of a two-ended cluster (in the set 2EP) were then removed from 1EP, and each contig in these 1EP clusters was aligned to the representative of the $2 \mathrm{EP}$ cluster(s) within 100 bases. If any $1 \mathrm{EP}$ contig in the cluster aligned with $\geq 80 \%$ coverage and $\geq 90 \%$ identity to the $2 \mathrm{EP}$ contig, the $1 \mathrm{EP}$ contig was added to the $2 \mathrm{EP}$ cluster.

We then added PopIns one-ended placement clusters to the right and left placements in 1EP (Supplementary Methods). Then for all clusters, we used NUCmer with default parameters to align contigs within each cluster to the representative $R$. If no alignment was found between a contig and $R$, the contig was removed from the cluster. We then realigned all other contigs to those in each of these filtered clusters, excluding contigs already determined to be part of a twoended insertion. Contigs $>99 \%$ identical over their whole length to any member of a cluster $C$ and covering at least $80 \%$ of the contig in $C$ were added to $C$. Contained, $99-100 \%$ identical contigs aligning with less than $80 \%$ coverage, were also included if they had at least five linking mates and at least $25 \%$ of those mates linked to within $5 \mathrm{~kb}$ of the placement location.

We then evaluated the one-ended placements to determine whether two contigs might belong to the same longer insertion, where one contig would 'fill' the left side of a gap and the other would fill the right side, possibly meeting in the middle. In some of these cases, the contigs might overlap, allowing us to merge them and create a single, longer insertion sequence. If placement positions were within 500 bases of one another, the sequences were aligned with NUCmer and merged if they were determined to be part of the same insertion (Supplementary Methods). Resultant merged sequences and their clusters were moved to the 2EP set (Fig. 1).

Finally, to remove any potential redundancy from placed clusters, we aligned all representatives from both one- and two-end placed clusters to one another (using nucmer -maxmatch - nosimplify) regardless of placement distance. If two representatives aligned with $\geq 98 \%$ identity, covering $\geq 95 \%$ of one of the contigs, and were placed within $5 \mathrm{~kb}$ of one another, these clusters were merged. To determine the representative (and therefore reported placement) of the merged clusters, two-ended placed representatives were favored over one-ended representatives, then our placements were preferred over PopIns, then longer contigs were favored over shorter contigs. By merging only placements within $5 \mathrm{~kb}$, we avoided merging contigs that were similar solely due to repetitive sequences but were unambiguously linked to different locations.

Unplaced contigs. For all unplaced contigs, we ran nucmer -maxmatch nosimplify with a minimum seed length of 31 (-1 31) and a minimum cluster size of 100 (-c 100) to align all contigs against one another. Contigs contained within another contig and aligning with $>95 \%$ identity were removed, and if contigs were annotated as identical by show-coords with $>97 \%$ identity, the smaller of the two was removed. If the ends of two contigs overlapped by at least 100 bases and a third contig was contained within the joined contigs, the contained contig was also removed. Trimming of up to 100 bases was permitted for finding overlaps. Finally, we aligned all resulting unplaced contigs to the placed representatives pre-trimming. If an unplaced contig aligned with $\geq 80 \%$ coverage and $\geq 90 \%$ identity, it was removed from the unplaced set, though it was not added into the placed cluster, as it did not meet the stricter placement or containment criteria used to create the clusters. 
In an additional attempt to place more contigs in the reference genome, we repeated the placement procedure described above, this time considering only the subset of linking mates that mapped to GRCh 38 with a mapping quality $>10$, and only attempting to place a contig if the contig end had a minimum of five such linking mates. This mapping quality criterion decreased the overall ambiguity of the putative locations for unplaced contigs (Supplementary Fig. 2); however, this additional placement effort only placed 150 additional contigs. We produced a file of putative linking locations for unplaced contigs by examining separately for each end the linking mates with a mapping quality $>10$. If $>50 \%$ of these high-quality linking mates for a given end pointed to the same region, where a region was defined by grouping mates within $2 \mathrm{~kb}$ of each other, we reported that region as the putative placement location for that end of the contig, as well as the total number of high-quality mates and the percentage of those mates linking to that location. For this report, the two contig ends were allowed to putatively link to different locations; in such cases both the start and end regions identified are provided, as these are the two most likely placement regions for the contig (Supplementary Table 3). The putative locations include high-copy repetitive sequences that may be underrepresented in GRCh38, and thus are overrepresented as linking locations (Supplementary Note 4 and Supplementary Fig. 3).

Additional screening and analyses. To screen for contaminants missed by Centrifuge, we used the Kraken metagenomics classifier ${ }^{36}$ on our final set of representative contigs to compare them to a database containing all complete bacterial and archaeal genomes, all viral genomes, selected fungi and protists, human, mouse, and known contaminant sequences. Any unclassified contig or contig hitting something other than mouse or human was further examined by running the blastn program ${ }^{26}$ to align the contig to NCBI's nonredundant nucleotide database. We removed all contigs (as likely contaminants) that had alignments to a non-chordate covering $>50 \%$ of the contig with a BLAST e-value $<10^{-10}$. We additionally removed a single contig, also an apparent contaminant, hitting Canis familiaris at $90 \%$ identity over the entire contig, but lacking any strong matches to primates. As expected, all of these contaminant contigs were found in the set of unplaced contigs. Deleted contaminants were examined for infections of interest, resulting in the incidental discovery of 29 individuals with malaria infections and 1 with human betaherpesvirus (Supplementary Note 5 and Supplementary Table 7).

To ensure the final set of contigs were truly absent from the human reference genome, we realigned all APG contigs to GRCh38.p10 using bwa-mem ${ }^{28}$ with default parameters. Two separate alignments were performed, one to the primary sequence and one to all patches and alternate loci. We removed any APG contigs with alignments to the primary assembly sequences at or above $90 \%$ identity over at least $80 \%$ of the contig's length, regardless of whether they had a better alignment to some alternate locus (Supplementary Methods). In Supplementary Tables 1 and 2, we report the best alignment location for each contig that had at least $50 \%$ of the contig aligned to GRCh38.p10 at $\geq 80 \%$ identity. All placed locations were intersected with the NCBI-provided gene annotations, GCF_000001405.36, which is the union of GenBank and RefSeq annotations for GRCh38.p10, and a translated BLAST search (blastx) was run against the comprehensive NCBI protein database to identify potential protein-coding regions in the APG sequences.

Calling presence/absence per sample. Raw contigs from the MaSuRCA assemblies (including contigs under $1 \mathrm{~kb}$ ) of all 910 individuals were aligned to the final set of APG contigs with bwa-mem using default parameters. Alignments to an APG contig aligning within $300 \mathrm{bp}$ of one another were chained to create longer alignments where possible. Identity of the chained alignment was taken to be the identity of these alignments weighted by length, and coverage was taken to be the total aligned bases over the total APG contig length. If an individual's raw contig alignments produced an alignment with $\geq 90 \%$ identity and $\geq 80 \%$ coverage to an APG contig, that APG contig was called as present, and a " 1 " was included in the matrix (Supplementary Data Set 1).

Additionally, for the placed contigs, because we had already determined which individuals contained these sequences, the genotype matrix was supplemented by adding a presence call (" 1 ") if we had determined that an individual had a contig in the placement cluster. This additional calling allowed increased sensitivity for individuals who had mate placement information available for the insertion, even when the contigs did not meet the identity/coverage criteria used for this presence/absence genotyping. The "genotype" matrix entries indicate presence/ absence calls represented as 1 or 0 ; heterozygous and homozygous genotypes are not differentiated.
To estimate whether the pan-genome would continue to grow as more individuals were sequenced, we randomly sampled varying numbers of individuals within our dataset and used the genotype matrix to determine, in each subset, how much of the APG sequence was present. Each data point was an average of ten random samplings, each with the same number of individuals. The amount of DNA added to the pan-genome appears to increase approximately linearly as the sample size grows, and has not reached an asymptote with 910 individuals (Supplementary Fig. 4).

We additionally called presence/absence of the APG insertions in 12 individuals from six European populations and 12 individuals from six African populations from the Simons Genome Diversity Project (Supplementary Table 5). We assembled these individual's contigs from raw read data via the same assembly pipeline used for the CAAPA data and then used the resulting MaSuRCA assembly contigs to make the presence/absence calls.

Comparisons to other genomes. We aligned all APG contigs to two additional genome assemblies: a Chinese genome assembly ${ }^{14}$ and a Korean genome assembly ${ }^{15}$. All alignments were performed using bwa-mem with default parameters. Because bwa-mem sometimes found multiple distinct alignments for a contig, the best query-consistent set of alignments for each contig was retained, so no part of an APG contig aligned to more than one location in the reference. The best query-consistent set was determined by comparing the sums of alignment length weighted by percent identity. We then filtered these alignments to these genomes, retaining alignments with an overall identity $\geq 90 \%$ that covered $\geq 80 \%$ of the contig.

We compared each APG contig's alignment(s) to the Chinese and Korean genomes to all alignments of the same contig to GRCh38.p10, including patches and alternate loci, obtained as previously described. Among the contigs aligning to the Chinese or Korean genomes, we examined further those with a better alignment (higher identity $\times$ coverage) to the Chinese or Korean genome than to GRCh38.p10. We separated these further into two categories, those contigs with a 'reasonably good' alignment to GRCh38.p10 ( $\geq 50 \%$ contig coverage and $\geq 80 \%$ identity for query-consistent sets of alignments within $1 \mathrm{~kb}$ of one another), and those lacking reasonably good alignments to GRCh38.p10.

Code availability. Commands and parameters are included in Supplementary Note 6. Custom scripts used are available upon reasonable request.

Reporting Summary. Further information on research design is available in the Nature Research Reporting Summary linked to this article.

\section{Data availability}

Raw sequence data used for this study are available from $\mathrm{dbGaP}$ with accession code phs001123.v1.p1. The African pan-genome contigs have been deposited at GenBank with accession code PDBU00000000. The version described in this paper is version PDBU01000000.

\section{References}

29. Langmead, B. \& Salzberg, S. L. Fast gapped-read alignment with Bowtie 2. Nat. Methods 9, 357-359 (2012).

30. Li, H. et al. The Sequence Alignment/Map format and SAMtools. Bioinformatics 25, 2078-2079 (2009).

31. Zimin, A. V. et al. The MaSuRCA genome assembler. Bioinformatics 29, 2669-2677 (2013)

32. Kim, D., Song, L., Breitwieser, F. P. \& Salzberg, S. L. Centrifuge: rapid and sensitive classification of metagenomic sequences. Genome Res. 26, 1721-1729 (2016).

33. Tarailo-Graovac, M. \& Chen, N. Using RepeatMasker to identify repetitive elements in genomic sequences. Curr. Protoc. Bioinformatics Chapter 4, Unit 4.10, (2009).

34. Delcher, A. L., Salzberg, S. L. \& Phillippy, A. M. Using MUMmer to identify similar regions in large sequence sets. Curr. Protoc. Bioinformatics Chapter 10, Unit 10.13, (2003).

35. Quinlan, A. R. \& Hall, I. M. BEDTools: a flexible suite of utilities for comparing genomic features. Bioinformatics $\mathbf{2 6}$ 841-842 (2010).

36. Wood, D. E. \& Salzberg, S. L. Kraken: ultrafast metagenomic sequence classification using exact alignments. Genome. Biol. 15, R46 (2014). 


\section{Life Sciences Reporting Summary}

Nature Research wishes to improve the reproducibility of the work that we publish. This form is intended for publication with all accepted life science papers and provides structure for consistency and transparency in reporting. Every life science submission will use this form; some list items might not apply to an individual manuscript, but all fields must be completed for clarity.

For further information on the points included in this form, see Reporting Life Sciences Research. For further information on Nature Research policies, including our data availability policy, see Authors \& Referees and the Editorial Policy Checklist.

\section{- Experimental design}

1. Sample size

Describe how sample size was determined.

N/A

2. Data exclusions

Describe any data exclusions.

N/A

3. Replication

Describe whether the experimental findings were reliably reproduced.

4. Randomization

Describe how samples/organisms/participants were allocated into experimental groups.

N/A

5. Blinding

Describe whether the investigators were blinded to group allocation during data collection and/or analysis.

Note: all studies involving animals and/or human research participants must disclose whether blinding and randomization were used.

6. Statistical parameters

For all figures and tables that use statistical methods, confirm that the following items are present in relevant figure legends (or in the Methods section if additional space is needed).

n/a $\mid$ Confirmed

\ $\square$ The exact sample size $(n)$ for each experimental group/condition, given as a discrete number and unit of measurement (animals, litters, cultures, etc.)

A description of how samples were collected, noting whether measurements were taken from distinct samples or whether the same

$\square$ sample was measured repeatedly

Х $\square$ A statement indicating how many times each experiment was replicated

$\triangle \square$ The statistical test(s) used and whether they are one- or two-sided (note: only common tests should be described solely by name; more

$\triangle \square$ complex techniques should be described in the Methods section)

Х $\square$ A description of any assumptions or corrections, such as an adjustment for multiple comparisons

Х $\square$ The test results (e.g. $P$ values) given as exact values whenever possible and with confidence intervals noted

$\bigotimes \square$ A clear description of statistics including central tendency (e.g. median, mean) and variation (e.g. standard deviation, interquartile range)

Х $\square$ Clearly defined error bars

See the web collection on statistics for biologists for further resources and guidance.

\section{- Software}

Policy information about availability of computer code

\section{Software}

Describe the software used to analyze the data in this 
For manuscripts utilizing custom algorithms or software that are central to the paper but not yet described in the published literature, software must be made available to editors and reviewers upon request. We strongly encourage code deposition in a community repository (e.g. GitHub). Nature Methods guidance for providing algorithms and software for publication provides further information on this topic.

\section{- Materials and reagents}

Policy information about availability of materials

\section{Materials availability}

Indicate whether there are restrictions on availability of unique materials or if these materials are only available for distribution by a for-profit company.

\section{Antibodies}

Describe the antibodies used and how they were validated for use in the system under study (i.e. assay and species).

10. Eukaryotic cell lines

a. State the source of each eukaryotic cell line used.

b. Describe the method of cell line authentication used.

c. Report whether the cell lines were tested for mycoplasma contamination.

d. If any of the cell lines used are listed in the database of commonly misidentified cell lines maintained by ICLAC, provide a scientific rationale for their use.

\section{N/A}

N/A

\section{N/A}

N/A

N/A

N/A

\section{- Animals and human research participants}

Policy information about studies involving animals; when reporting animal research, follow the ARRIVE guidelines

\section{Description of research animals}

Provide details on animals and/or animal-derived materials used in the study.

Policy information about studies involving human research participants

\section{Description of human research participants}

Describe the covariate-relevant population 